This item was submitted to Loughborough's Research Repository by the author.

Items in Figshare are protected by copyright, with all rights reserved, unless otherwise indicated.

\title{
Inertial frequency response provided by battery energy storage systems:
} probabilistic assessment

PLEASE CITE THE PUBLISHED VERSION

https://doi.org/10.1109/ICCEP.2017.8004847

PUBLISHER

(c) IEEE

VERSION

AM (Accepted Manuscript)

LICENCE

CC BY-NC-ND 4.0

\section{REPOSITORY RECORD}

Gonzalez-Longatt, Francisco M., Samir M. Alhejaj, Andrea Bonfiglio, Renato Procopio, and Jose L. Rueda. 2019. "Inertial Frequency Response Provided by Battery Energy Storage Systems: Probabilistic Assessment". figshare. https://hdl.handle.net/2134/25772. 


\title{
Inertial Frequency Response provided by Battery Energy Storage Systems: Probabilistic Assessment
}

\author{
F Gonzalez-Longatt*, S. Alhejaj*, A. Bonfiglio**, R. Procopio**, and J.L. Rueda*** \\ * CREST, Loughborough University, United Kingdom \\ ** University of Genoa - DITEN, Italy \\ *** Delft University of Technology, The Netherlands
}

\begin{abstract}
This paper proposes a methodology for assessment to measure the impact of the inertial frequency response provided by battery energy storage systems (BESS) considering power system uncertainties. The proposed methodology is used to assess the impact of the BESS equipped with sensible frequency controller on the frequency behaviour of the system after a system frequency disturbance considering the variability coming from power system uncertainties. The proposed meteorology is tested in an illustrative power system considering the integration of a grid-scale BESS equipped with inertial frequency response controller, the uncertainty of the power system is simulated by the variability of the power demand. Monte Carlo simulations and time-domain simulations, using DIgSILENT $^{\circledR}$ PowerFactory $^{\mathrm{TM}}$ are combined in the implementation of the proposed methodology. Simulation results demonstrate the suitability of the proposed approach to obtain probabilistic distribution functions (PDF) of the main system frequency response.
\end{abstract}

Index Terms-- Battery energy storage system, frequency response, Monte-Carlo Simulation, probabilistic assessment.

\section{INTRODUCTION}

Power system like other systems that have a real world phenomenon has a number of possible randomnesses due to many events and scenarios that are involved. In order to model such as a system, researchers sometimes follow probabilistic or stochastic approaches [1] to predict the results or use these approaches to get a better-optimized design for the system. In general, probabilistic approaches are used in many risk assessments since they quantify the amount of variation and uncertainty compare to the deterministic methods that provide an only fixed set of values. Therefore, implementing these approaches make it more possible to search for the values of interest within the specified space of the search. The core principle is to use distributions instead of fixed values to measure and quantify the data for variables that mostly affect the system behaviour and create the uncertainty. Mainly, distribution defines the range of possible values and shows which value within the expected range. In turn, this will provide a better foundation for making a decision about the best possible solutions or insecticide risks.

Many probabilistic approaches are used in power system analysis to assess the different aspects of the risks [2], reliability [3], security [4] and stability [5]. However, the majority probabilistic assessments published researchers for power system stability are written to discuss mainly small signal transient and voltage stability. However, probabilistic approaches can also be used to analysis frequency stability based on the randomness of load and intermittency of power generation supplied by the wind and solar with many associated uncertain events. They also provide an outlook for optimisation techniques that are required to control the frequency response for the ancillary services. Therefore, random and discrete events could be well analysed by the statistical approach and with assistance from the use of stochastic data model.

This paper proposes a methodology for assessment to measure the impact of the inertial frequency response provided by BESS considering power systems uncertainties.

The paper is organised as the following: Section II introduces the proposed methodology of probability assessment and describes the probabilistic model used for the power system uncertainties. Section III presents numerical results of the implementation of the proposed methodology using a pedagogic test system. Finally, Section IV presents the main findings and conclusions.

\section{I.MODELLING OF BATTERY ENERGY STORAGE SYSTEM (BESS)}

There are several technologies available for Electrical Energy Storage System (EESS), some of them used a classical three-step process. The core of the energy storage system is the transformation of electrical energy into some other energy form that could be reconverted into electricity [6]. In this paper, the EESS consists of a classical battery energy storage system (BESS) -see Fig 1. A very generic model of a BESS consists of two main subsystems [6, 7]: (i) a power conversion system (PCS) and the battery energy system (BES).

The power conversion system uses bi-directional AC/DC converter (inverter/rectifier) as the main interface between the BES and the power grid. The PCS is used to transform the DC-voltage from the BES into AC-voltage conditions required by the power grid [8]. A set of controllers are included in the PCS; those control loops are designed to enable specific functionalities interfacing the BES and the power network. The main modelling details of those subsystems are presented in the next subsections. 


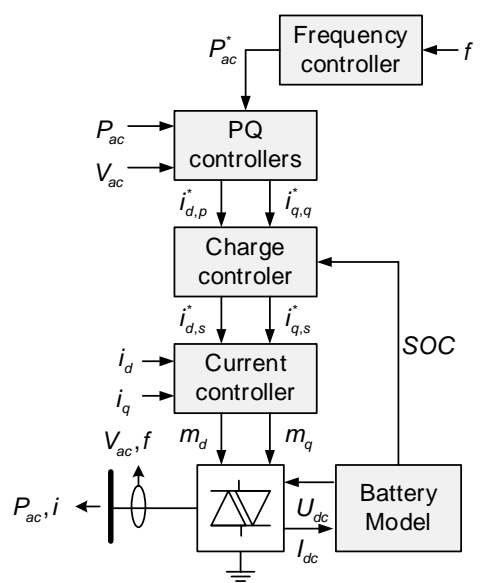

Fig. 1. A representative block diagram of a Battery Energy Storage System (BESS) [8].

\section{A. Model of the Power conversion system (PCS)}

This paper is focused on the system frequency response, as a consequence, the main attention is on the control behaviour of ac/dc PWM-converter instead of switching frequencies, or high frequencies phenomenon. Taking into account the previous considerations, the fundamental frequency model is used in this paper in order to model the two-level PWM converter which operated in a stator voltage oriented $d q$ reference frame. $d$-axis represents the active and $q$-axis the reactive component [9].

The line-line AC voltage (rms value) is described based on $d q$ reference frame as:

$$
V_{a c}=V_{d}+j V_{q}
$$

where the $d$ and $q$ axis component of the ac voltage are related to the dc voltage $\left(U_{d c}\right)$ :

$$
V_{d}=\frac{\sqrt{3}}{2 \sqrt{2}} m_{d} U_{d c} \quad V_{q}=\frac{\sqrt{3}}{2 \sqrt{2}} m_{q} U_{d c}
$$

where $m_{d}$ and $m_{q}$ are the real and imaginary part of the modulation index:

$$
m=m_{d}+j m_{q}
$$

\section{A. Model of the Battery Energy System (BES)}

The BES uses reversible electrochemical reactions to convert/store electricity. There are several batteries technologies commercially available in the market [6]: Lead-acid batteries (Pb-acid), Lithium-ion batteries (Liion), Nickel-cadmium batteries (NiCd), molten salt batteries like sodium-sulfur battery (NaS), aluminiumion (Al-ion), vanadium redox battery (VRB), liquid metal batteries, Sodium-ion batteries (SIB).

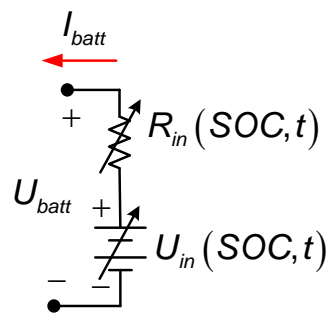

Fig. 2. Simple equivalent circuit representative of a typical electrochemical battery $[8,10]$.
Batteries using $\mathrm{Pb}$-acid provide a scalable technology base for providing short-term storage, in particular, frequency control. Modelling the battery is one of the most challenging situations in the energy storage system. However, since the battery is an electric bipole, were it linear, its more natural model would be constituted by an electromotive force $\left(U_{i n}\right)$ in series with an internal impedance $\left(R_{i n}\right)$, both function of time $(t)$.

In this paper, the simple battery model is shown in Fig. 2 is used. The state of charge (SOC) is calculated using an integrator which takes into account the current of the battery $\left(I_{\text {batt }}\right)$ :

$$
U_{d c}=U_{\text {max }} S O C+U_{\text {max }}(1-S O C)-I_{\text {batt }} Z_{i}
$$

where $U_{\min }$ represents the cell voltage discharged cell (V), $U_{\max }$ is the maximum voltage of the battery cell (V).

\section{B. Model of the battery charge controller}

The charge controller consists of two parts (Fig. 3): (i) Charging logic to achieve the SOC boundary conditions $\left(S O C_{\min } \leq S O C \leq S O C_{\max }\right)$, and (ii) current limiter to limits the absolute value of the current order according to limits $\left(I_{\min } \leq i \leq I_{\max }\right)$. The $d$-axis current always has the higher priority than the $q$-axis current. The signal $\Delta i$ is the difference of the reference $d$-axis current from the $P Q$ controller and $\left(i_{d, p}^{*}\right)$ the modified $d$-current from the charging logic $\left(i_{d, s}^{*}\right)$. The feedback of that signal to the $P Q$-controller prevents a windup of the PI-controller.

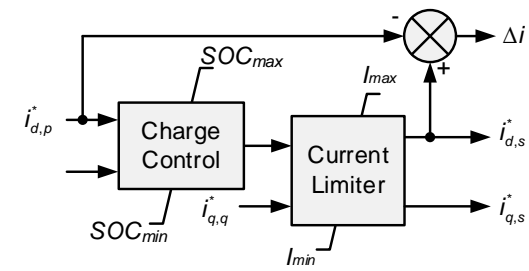

Fig. 3. Block diagram of the battery charge controller [7].

\section{Model of the current controller}

The input currents to the controller are the converter's AC-currents expressed in a reference $d q$ frame $\left(i_{d}, i_{q}\right)$. The output signals $m_{d}$ and $m_{q}$ are defined in the same reference frame and transformed back to a global reference frame using the same reference angle. A proportional-integral (PI) control loop is used to regulate the $d$ and $q$-axis current components $\left(i_{d}, i_{q}\right)$ based on a PI controller regulating the battery charge; these are shown in Fig. 4.
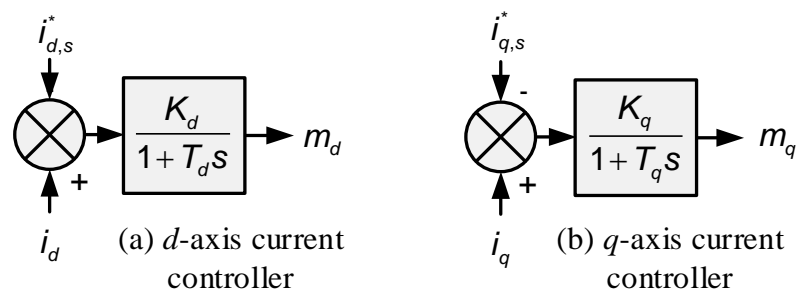

Fig. 4. Block diagram of the current controllers [8].

\section{Model of the PQ-Controller}

The controller for the active and reactive power is 
shown in Fig 5. The voltage (or $Q$ ) controller has a very slow current controller for set point tracking and a slope with a dead band for proportional voltage support.

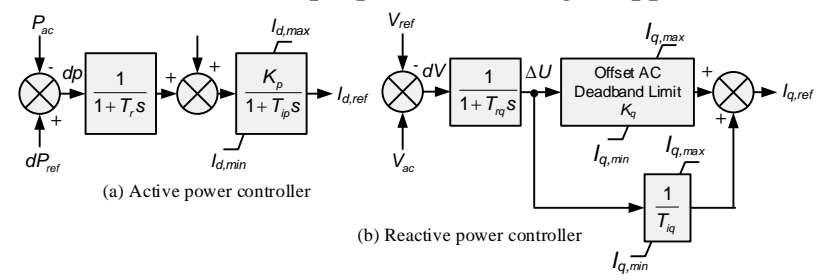

Fig. 5. Block diagram of the $P Q$-Controller.

\section{B. Model of the Frequency Controller}

During a system frequency disturbance (SFD) the generation/demand power balance is lost, the system frequency will change at a rate initially determined by the total system inertia $\left(H_{T}\right)$ [11]. Power sources and EESS connected to the grid using full-rated power converters has the potential to provide a very fast frequency response. Several names have been using in the wind turbine industry to define the controllers to enable a power converter to mimic the inertial response of a synchronous generator [12]: Artificial, Emulated, Simulated, or Synthetic Inertia.

The synthetic inertia concept allows a controller to the take the kinetic energy from the rotating mass in a wind turbine generator (WTG) [13]. This concept can be perfectly applied to BESS, but instead of taking kinetic energy from the rotating masses, the controller enables to discharge the battery in a controlled way producing an additional power in the form of inertial power.

The synthetic inertia controller can be understood as a simple loop that increases the electric power output of the PCS during the initial stages of a significant downward frequency event. The inertial power or power produced during the system frequency disturbance is calculated using the equivalent to the swing equation of a synchronous generator [14]:

$$
\Delta P=2 f H_{s y n} \frac{d f}{d t}
$$

where $H_{\text {syn }}$ represent the value of the synthetic inertia (sec) and $f$ is system frequency (p.u). Implementation of synthetic inertia controller is depicted in Fig. 6, where $P^{*}{ }_{a c}=\Delta P$.

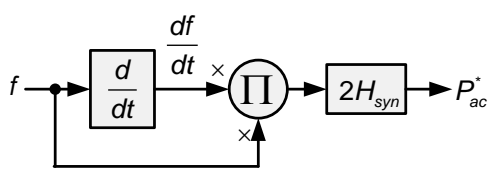

Fig. 6. Block diagram of the of the frequency controller [15]

It is important to point out the synthetic inertia $H_{\text {syn }}$, represent the gain of the proportional controller in the frequency controller. The gain of the frequency controller could be any value when the inertia controller is installed in WTG; the gain takes values related with the physical inertia constant of the rotating components. However, when the concept of inertia controller is applied to BESS, the physical understanding is lost, and as a consequence, any value could be used but consideration about the rate of discharge of the battery must be taken into account. A sudden high discharge current could lead to excessive heat build-up and thermal runaway.

\section{Proposed Probabilistic Assessment}

The appropriate analysis of power system considered increased number of uncertainties requires a different approach to the classical deterministic. Modelling the uncertainties using stochastics models has been the roots of the probabilistic approaches. The Stochastic Power Flow (SPF) analysis is one successful example of that. In the SPF the power generation and the grid configurations are both considered as discrete random variables, while the load demand is considered as a continuous random variable [16]. The aim of the PSF is to create Probabilistic Density Functions (PDF) to describe the performance of the power system.

The increased levels of uncertainties in power system has special impact on the power system dynamic, as a consequence, the probabilistic assessment has been a tendency in recent years. However, power system dynamic is a complex problem in terms of size and nonlinearity and coping with them require ingenious approaches. This paper proposes a methodology for assessment to measure the impact of the inertial frequency response provided by BESS considering power systems uncertainties. The core of the proposed methodology is the use of robust uncertainties modelling considering stochastic properties and scenarios generation and the use of a widely used numerical approach of probabilistic assessment, the Monte Carlo (MC) method.

The proposed methodology consists of the following sequence of steps: (i) Uncertainties modelling, (ii) Scenarios creation, (iii) Monte Carlo Simulation, (iv) Probabilistic analysis of the results.

\section{Uncertainties Modelling}

Uncertainty is a very complex concept; however, it can be described as a lack of knowledge regarding the true value of a parameter. The mathematical model that describe the uncertainties is a delicate component of the proposed methodology.

A stochastic model can be used to represent uncertainties, the model used for each uncertainty is critical for the proposed approach. The uncertainty model should be able to reproduce the associated variability systematically. The uncertainties in the future electrical power system can come from so many different sources: generation, demand, etc.

The first step is to select the relevant uncertainties related to the phenomenon of interest. This methodology is interested in the dynamic system frequency response, as a consequence, any source of power imbalance is considered the universe of potential uncertainties. The stochastic model of the uncertainties can be defined by a continuous of discrete probabilistic distribution with 
known or unknown parameters. Many distribution models are proposed to create probabilistic models based on the distribution function and the type of data that are needed. The most popular of these distribution models are uniform, binomial, Passion and Gaussian distribution.

In this paper, the uncertainty of the system inputs are modelled considering stochastic nature of the system loads. The uncertainty coming from changes in the electricity demand can be modelled using a stochastics model where the powder demand is assumed to be the random variable $\left(P_{L}\right)$.

The literature is well-documented with scientific documents dealing with the specific details of the load model, but the majority agrees the uncertainty related to the loads can be assumed normally distributed, as a consequence, a simple two-parameter Gaussian distribution can be used.

The load is assumed to be a random variable $\left(P_{L, i}\right)$ normally distributed within each hour for a given time period [17]. Then, the probability distribution function (PDF) of the power demand of the $i-$ th load $\left(P_{L, i}\right)$ is given by the following expression:

$$
f_{P_{L, i}}\left(P_{L, i}\right)=\frac{1}{\sigma_{i} \sqrt{2 \pi}} e^{-\frac{\left(P_{L, i}-\mu_{L, i}\right)^{2}}{2 \sigma_{i}^{2}}}
$$

where $\mu_{L, i}$ is the mean value of the electric demand and $\sigma_{i}$ is the standard deviation. Using this approach, the uncertainty related to a power demand is defined by two main parameters: $\mu_{L, i}$ and $\sigma_{i}$. Assuming the power demand follow a Gaussian distribution in a typical load is a reasonable assumption that makes sense based on the bets citify knowledge.

This approach can be easy extended to represents any other source of uncertainty in future power systems. The main difference on modelling other sources of uncertainties come from the model assumptions, e.g. probabilistic distribution and number of parameters. In general sense, the best uncertainty model has the following characteristics: simple, realistic, efficient, useful, reliable, valid, etc. Literature is rich in more detailed models for some other sources of uncertainties as wind speed [16] and wind power [18], classical generation, more complex loads [19]

\section{Scenarios creation}

The random variables are used to represent variables whose values cannot be predicted with certainty. If a random process is considered stationary that means the statistical attributes of the process are not changing - and if there is no serial correlation in the spatial or temporal sequence of observed values. Single probability distributions can describe the previously described random processes as the uncertainties model presented in the previous subsection. The use of parametric PDFs description of the uncertainties allows the creation of scenarios based on random sampling; the created scenarios then are used by the Monte Carlo method to obtain numerical results. The stochastic models, for the considered uncertainties, are used to create a set of scenarios. Random numbers are used in the stochastics models in order to create samples for each uncertainty, and then the samples of the forecasted uncertainties are combined together to create scenarios.

The inverse transform sampling (also known as Smirnov transform) is used in this paper for scenarios creations.

The power demand uncertainty is represented by the random variable $p_{L, i}$, whose probabilistic distribution can be characterised by the cumulative distribution function (cdf) $F_{\mathrm{pLi}}$. Now, in order to generate values of $p_{L, i}$ which are distributed according to cdf, the inverse transform sampling method is used: (i) generate a random number $p$ from the standard uniform distribution in the interval $p \in[0,1]$; (ii) compute the value p such that $F_{P L i}(p)=\mathrm{u}$; (iii) take $p$ to be the random number drawn from the distribution described by $F_{p L i}$.

\section{E. Monte Carlo Simulations (MSC)}

The Monte Carlo methods or Monte Carlo experiments are the core of the proposed methodology. MSC uses the deterministic model of the system and systematically solved it considering each scenario.

In this paper, the main interest is the system frequency response. The best way to obtain the deterministic system frequency response of the power system after a disturbance is by using time-domain simulations. This approach also, allows an easy implementation of the proposed methodology by using a commercially available power system analysis software for to obtain the system frequency response.

The Monte Carlo method uses time-domain simulation considering each scenario, and then collect the deterministic data of each scenario for the most relevant variables of the system frequency response are collected.

Several performance indicators are available to evaluate the performance of the system frequency response (SFR): (i) Maximum frequency gradient $\left([d f / d t]_{\max }\right)$ as observed by ROCOF (Rate-Of-Change-OfFrequency) relays; (ii) Maximum frequency deviation $\left(f_{\text {max }}\right)$ as observed by under-frequency relays. It is defined as the absolute frequency deviation from the nominal frequency $\left(f_{n}\right)$. (iii) Frequency nadir $\left(f_{\min }\right)$ measures the minimum post-contingency frequency; (iv) Frequency nadir time $\left(t_{\min }\right)$ is the time it takes for the response to reach $f_{\min }$; (v) Quasi-steady-state deviation $\left(\Delta f_{s s}\right)$ is the deviation between $f_{n}$ and the final value $\left(f_{s s}\right)$. In this paper, the main indicators used are $f_{\min }$ and ROCOF.

The time-domain simulation is a time-consuming process, and it generates a considerable volume of result data, it must be post-processed in order to extract the appropriated information for the probabilistic assessment and the impact of the inertial frequency response provided by battery energy storage systems (BESS) considering power system uncertainties. 


\section{F. Probabilistic Analysis of the Results}

The solution of the deterministic model is performed per each scenario, the solution of the time-domain simulation is a set of time-series for each variable of interest. The MCS will solve the deterministic model for each sample, creating at the end a massive volume of information to be processed. In this paper the main interest is the system frequency response, as a consequence, specific indicators are defined and collected for each time-series: $f_{\min }$ and ROCOF.

In the proposed approach the collected data is collected from the time-domain simulation, and it is used to create a set of time series of the generator's frequency. The frequency of centre inertia is used to combines the generator's frequency in a single time-series, and it could be processed to obtain frequency indicators as minimum frequency, the maximum rate of change of frequency $\left([d f / d t]_{\max }\right)$, steady-state frequency deviation, etc. The set of indicators obtained considering all the scenarios are used to create probability distributions for each variable. The process of creating the PDF's from the set of indicators is explained in the final paper.

\section{SimULATION AND RESULTS}

The proposed a methodology for assessment to measure the impact of the inertial frequency response provided by BESS considering power systems uncertainties is tested in this section. The proposed methodology is tested and illustrated using a numerical example. A simple test system is used (see Fig. 1). It consists of two synchronous generators $\left(P_{n}=400 \mathrm{MVW}\right.$, $H=5.0 \mathrm{sec}$ ) connected to an equivalent transmission system, where a $50 \mathrm{MW}$ BESS in connected. The synchronous generator is equipped with GAST governor and IEEE Type I AVR [20]. The full model of the BESS has been developed using DigSILENT Simulation Language (DSL) consider the previous section models, and the BESS has been enabled to provide system frequency response using an inertia frequency response controller.

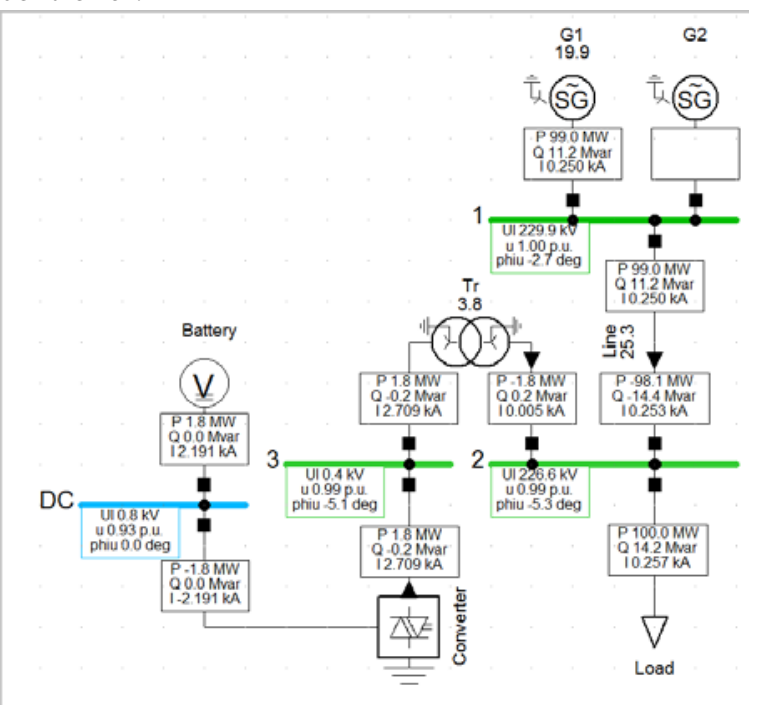

Fig. 7. Illustrative two-machines+BESS test system.
For illustrative purposes, a single source of power system uncertainty is considered. The uncertainties coming from the demand are modelled using a Gaussian distribution $\left(\mu_{P L}=100 \mathrm{MW}, \sigma_{P L}= \pm 10 \%\right.$ ). Details of the discrete probability distribution of a 1,000 samples power demand are shown in Fig. 8.

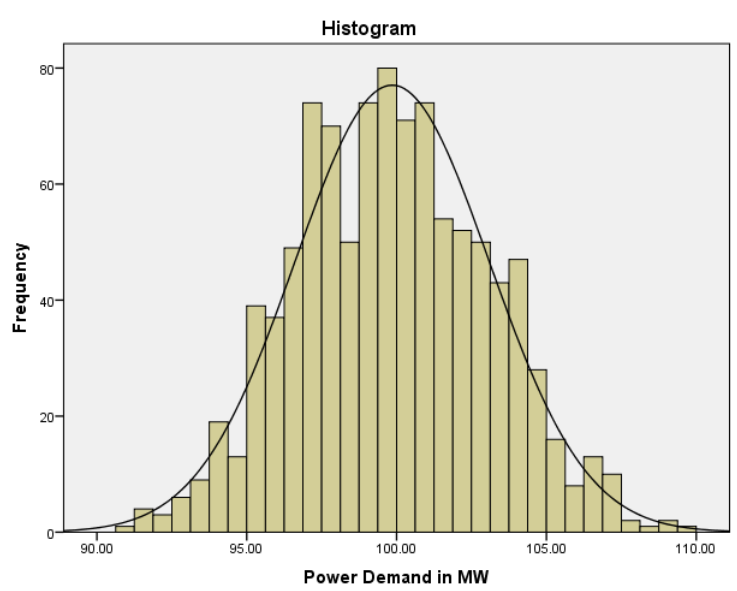

Fig. 8. The probability distribution of the simulated load in MW.

The system frequency response of the test system is analysed using time-domain simulations. The power system analysis software DIgSILENT PowerFactory is used to obtain the system frequency response, controls model of all components are included, and the user defined model is used in the BESS. The system frequency disturbance consists of sudden disconnection of Generator G2 (see Fig. 7). Previous to the disturbance the G1 is considered as reference machine and the G2 is dispatched to cover $50 \%$ of the total system load $\left(P_{L}\right)$. Two main simulation cases are studied in this section: Case I: Without BESS and Case II: With BESS. The Case I is selected as reference and Case II is designed to measure the impact of using BESS for inertial frequency response.

A DIgSILENT Simulation Programming (DPL) script is developed to automatize the time-domain simulations. The DPL script read the power demand samples from a MS Excel file and run the RMS time-domain simulation, and time series of relevant variables are exported in text format for later post-processing. The DPL script runs 1,000 time-domain simulations; it is a time-consuming process.

A MATLAB script was developed to post-processing the results of the Monte Carlo simulations. The MATLAB program read the text files and extract the relevant information and calculates the indexes for frequency response: $R O C O F$ and $f_{\text {min }}$.

Table I shows the main statistic indicators for the frequency indicator are the studied cases.

Fig. 9 and 10 show the discrete probabilistic distribution function of the minimum frequency and ROCOF for the Case I and II respectively.

The sudden disconnection of G2 produces a power imbalance $\left(\triangle P=50 \% P_{L}\right)$; the BESS is able of providing 
an inertial response, and however, the effect on the minimum frequency $\left(f_{\min }\right)$ is minimum. It is an expected conclusion because the inertial controller aims to improve the ROCOF. However, this results shows how the initial state of the power system (generation/demand) affect the minimum frequency. The average minimum frequency is $49.78 \mathrm{~Hz}$, and the minimum frequency is below 49.715 $\mathrm{Hz}$ in $67 \%$ of the cases.

TABLE I. BATTERY MODELS PARAMETERS

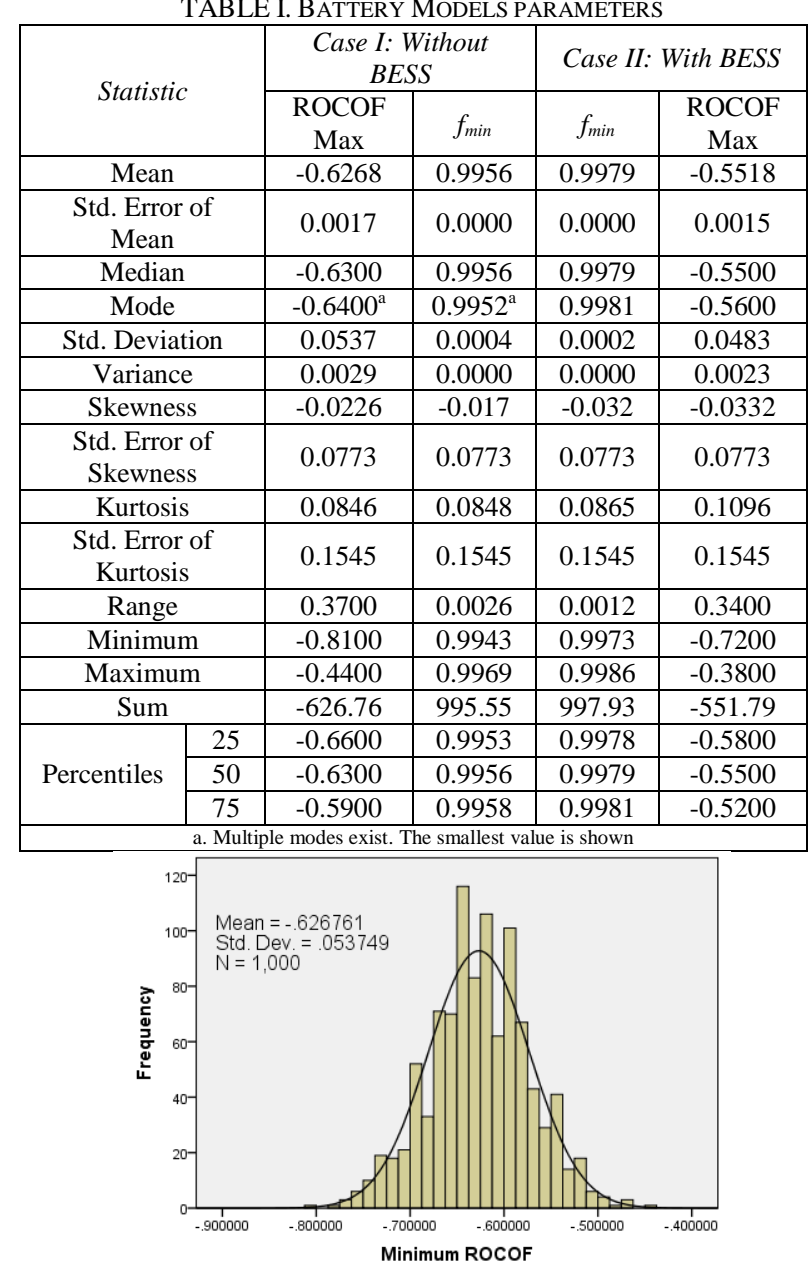

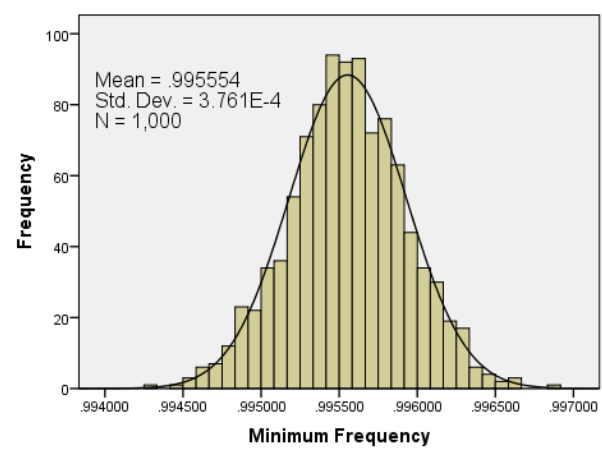

Fig. 9. PDF of $f_{\min }$ and ROCOF: Case I: Without BESS.

The main impact of using BESS to provide inertial response, as expected, is on the ROCOF. Case I shows a high average ROCOF of $-0.6268 \mathrm{pu} / \mathrm{sec}$, but when the BESS is enabled to provide frequency support the ROCOF is reduced to $-0.5518 \mathrm{pu} / \mathrm{sec}$. Also, the BESS help to control the system frequency, and it is evident with the reduction in the standard deviation of the ROCOF on the 1,000 simulated cases.
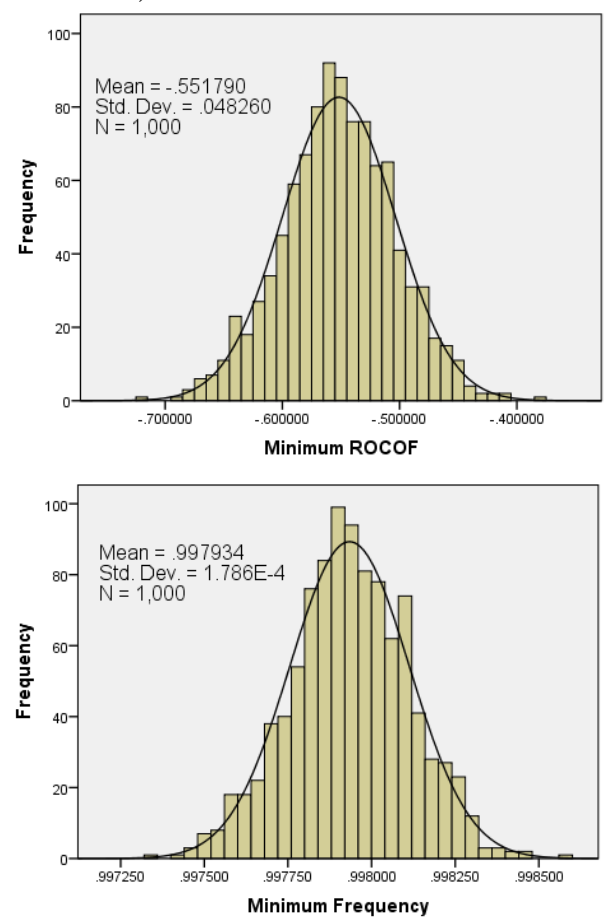

Fig. 10. PDF of $f_{\min }$ and ROCOF: Case II: With BESS.

\section{CONCLUSIONS}

This paper proposes a methodology for assessment to measure the impact of the inertial frequency response provided by battery energy storage systems (BESS) considering power system uncertainties. The core of the methodology is the combination of Monte Carlo simulations and time-domains simulations. The proposed methodology is used to assess the impact of the BESS equipped with sensible frequency controller on the frequency behaviour of the system after a system frequency disturbance considering the variability coming from power system uncertainties. Simulations results demonstrate the suitability of the proposed approach.

\section{APPENDIX}

TABLE A. BATTERY MODELS PARAMETERS

\begin{tabular}{l|c|c|c}
\hline \multicolumn{1}{c|}{ Description } & Parameter & Unit & Value \\
\hline State of change & SOC & - & 0.8 \\
Single Cell Capacity & $W_{n}$ & $\mathrm{Ah}$ & 1.2 \\
Min. Voltage of empty cell & $U_{\min }$ & $\mathrm{V}$ & 12.00 \\
max. Voltage of full cell & $U_{\max }$ & $\mathrm{V}$ & 13.85 \\
Number of parallel connected cells & $N_{p}$ & - & 60 \\
Number of parallel connected cells & $N_{s}$ & - & 65 \\
Nominal BESS Voltage & $U_{n}$ & $\mathrm{~V}$ & 900 \\
Internal Resistance per cell & $Z_{i}$ & $\Omega$ & 0.001 \\
\hline
\end{tabular}

TABLE B. BATTERY CHARGER CONTROLLER PARAMETERS

\begin{tabular}{l|c|c|c}
\hline \multicolumn{1}{c|}{ Description } & Parameter & Unit & Value \\
\hline Min charge current & Imin & p.u. & 0.1 \\
Min state of charge & SOC $_{\min }$ & p.u. & 0.0 \\
Max state of charge & SOC $_{\max }$ & p.u. & 1.0 \\
Max absolute current & $I_{\max }$ & p.u & 1.0 \\
\hline
\end{tabular}


TABLE C. CURRENT CONTROLLER PARAMETERS

\begin{tabular}{l|c|c|c}
\hline \multicolumn{1}{c|}{ Description } & Parameter & Unit & Value \\
\hline Proportional gain, $d$-axis & $K_{d}$ & - & 0.1 \\
Integration time constant, d-axis & $T_{d}$ & sec & 0.001 \\
Proportional gain, $q$-axis & $K_{q}$ & - & 0.1 \\
Integration time constant, q-axis & $T_{q}$ & sec & 0.001 \\
\hline
\end{tabular}

TABLE D. CURRENT CONTROLLER PARAMETERS

\begin{tabular}{l|c|c|c}
\hline \multicolumn{1}{c|}{ Description } & Parameter & Unit & Value \\
\hline Filter time constant, $d$-axis & $T_{r}$ & sec & 0.05 \\
Filter time contact, $q$-axis & $T_{r q}$ & sec & 0.01 \\
Proportional gain, $d$-axis & $K_{p}$ & - & 2.00 \\
Integration time constant, d-axis & $T_{d}$ & sec & 0.10 \\
Deadband for proportional gain & $K_{d b}$ & - & 0.10 \\
Proportional gain, q-axis & $K_{q}$ & - & 2.00 \\
Integrator time constant, q-axis & $T_{q}$ & sec & 1.00 \\
Min. current, $d$-axis & $I_{d \min }$ & p.u. & -1.00 \\
Min. current, $q$-axis & $I_{q \min }$ & p.u. & 1.00 \\
Max. current, $d$-axis & $I_{d \max }$ & p.u. & -1.00 \\
Min. current, $q$-axis & $I_{q \max }$ & p.u. & 1.00 \\
\hline
\end{tabular}

\section{REFERENCES}

[1] R. Allan and R. Billinton, "Probabilistic assessment of power systems," Proceedings of the IEEE, vol. 88, no. 2, pp. 140-162, 2000.

[2] W. Li, Risk Assessment of Power Systems:Models, Methods, and Applications. Wiley-IEEE Press, 2014.

[3] M. T. Schilling, J. C. S. d. Souza, and M. B. D. C. Filho, "Power System Probabilistic Reliability Assessment: Current Procedures in Brazil," IEEE Transactions on Power Systems, vol. 23, no. 3, pp. 868-876, 2008.

[4] K. Morison, W. Lei, and P. Kundur, "Power system security assessment," IEEE Power and Energy Magazine, vol. 2, no. 5, pp. 30-39, 2004.

[5] P. M. Anderson and A. Bose, "A Probabilistic Approach to Power System Stability Analysis," IEEE Transactions on Power Apparatus and Systems, vol. PAS-102, no. 8, pp. 2430-2439, 1983.

[6] F. M. Gonzalez-Longatt and S. M. Alhejaj, "Enabling inertial response in utility-scale battery energy storage system," in 2016 IEEE Innovative Smart Grid Technologies - Asia (ISGT-Asia), 2016, pp. 605-610.

[7] S. M. Alhejaj and F. M. Gonzalez-Longatt, "Impact of inertia emulation control of grid-scale BESS on power system frequency response," in 2016 International Conference for Students on Applied Engineering (ICSAE), 2016, pp. 254-258.

[8] S. Alhejaj and F. Gonzalez-Longatt, "Investigation on gridscale BESS providing Inertial Response Support," presented at the IEEE PES POWERCON 2016, Wollongong Australia, 28 September - 1 October 2016, 2016.

[9] M. Deepak, R. J. Abraham, F. M. Gonzalez-Longatt, D. M. Greenwood, and H.-S. Rajamani, "A novel approach to frequency support in a wind integrated power system," Renewable Energy, vol. 108, pp. 194-206, 8// 2017.
[10] M. Ceraolo, "New dynamical models of lead-acid batteries," IEEE Transactions on Power Systems, vol. 15, no. 4, pp. 1184-1190, 2000.

[11] A. Bonfiglio, F. Gonzalez-Longatt, and R. Procopio, "Integrated Inertial and Droop Frequency Controller for Variable Speed Wind Generators," WSEAS Transactions on Environment and Development, vol. 12, no. 18, pp. 167177, 2016.

[12] F. Gonzalez-Longatt, "Impact of emulated inertia from wind power on under-frequency protection schemes of future power systems," (in English), Journal of Modern Power Systems and Clean Energy, pp. 1-8, 2015/08/12 2015.

[13] A. Bonfiglio, F. Delfino, F. Gonzalez-Longatt, and R. Procopio, "Steady-state assessments of PMSGs in wind generating units," International Journal of Electrical Power \& Energy Systems, vol. 90, pp. 87-93, 9// 2017.

[14] F. Gonzalez-Longatt, "Frequency Control and Inertial Response Schemes for the Future Power Networks," in Large Scale Renewable Power Generation, J. Hossain and A. Mahmud, Eds. (Green Energy and Technology: Springer Singapore, 2014, pp. 193-231.

[15] F. Gonzalez-Longatt, A. A. Bonfiglio, R. Procopio, and B. Verduci, "Evaluation of Inertial Response Controllers for Full-Rated Power Converter Wind Turbine (Type 4)," presented at the IEEE PES General Meeting 2016, Boston, USA, 17-21 July 2016, 2016.

[16] F. Gonzalez-Longatt, J. L. Rueda, and D. Dogdanov, "Assessment of the Variability on the Electricity Production of Wind Turbines Technologies considering Time-Series of Wind Speed," presented at the Efficacité énergétique - sources d'énergies renouvelables - protection de l'environnement COFRET'12, Sozopol, Bulgaria, 6-8 September 2013, 2012.

[17] N. D. Hatziargyriou, T. S. Karakatsanis, and M. Papadopoulos, "Probabilistic load flow in distribution systems containing dispersed wind power generation," Power Systems, IEEE Transactions on, vol. 8, no. 1, pp. 159-165, 1993.

[18] F. Gonzalez-Longatt, J. L. Rueda, and D. Bogdanov, "Probabilistic assessment of operational risk considering different wind turbine technologies," in 3rd IEEE PES International Conference and Exhibition on Innovative Smart Grid Technologies (ISGT Europe 2012), 2012, pp. 16.

[19] F. M. Gonzalez-Longatt, J. L. Rueda, I. Erlich, D. Bogdanov, and W. Villa, "Identification of Gaussian mixture model using Mean Variance Mapping Optimization: Venezuelan case," in 3rd IEEE PES International Conference and Exhibition on Innovative Smart Grid Technologies (ISGT Europe 2012) 2012, pp. 16.

[20] A. Bonfiglio, F. Delfino, M. Invernizzi, A. Perfumo, and R. Procopio, "A Feedback Linearization Scheme for the Control of Synchronous Generators," Electric Power Components and Systems, vol. 40, no. 16, pp. 1842-1869, 2012/10/15 2012. 